\title{
Screening ionic solvents for enhancing the solubility of water-insoluble natural dyes
}

Luiz Fernando Lepre ${ }^{1}$, Stéphane Sabelle ${ }^{2}$, Floriane Beaumard ${ }^{2}$, Ann Detroyer ${ }^{2}$, MarieCéline Frantz ${ }^{2}$, Agilio Padua ${ }^{1}$, Margarida Costa Gomes ${ }^{1,{ }^{*}}$

${ }^{1}$ Laboratoire de Chimie, École Normale Supérieure de Lyon \& CNRS, 69364 Lyon, France.

2 L'Oréal Research \& Innovation, 1 av. Eugène Schueller, 93600 Aulnay-sous-Bois, France.

*margarida.costa-gomes@ens-lyon.fr

\section{Supporting Information}


Structures of the cations and anions studied in this work

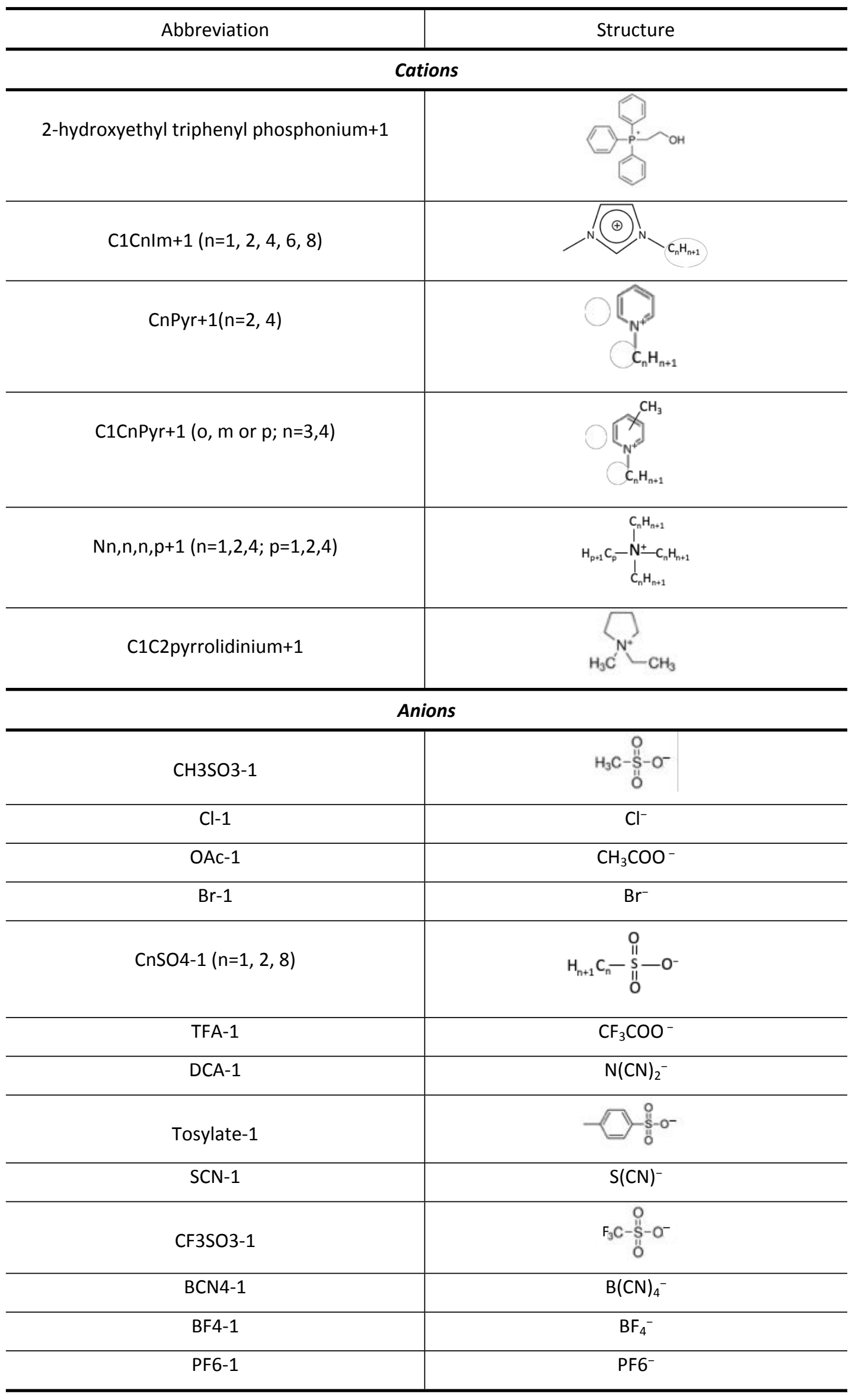



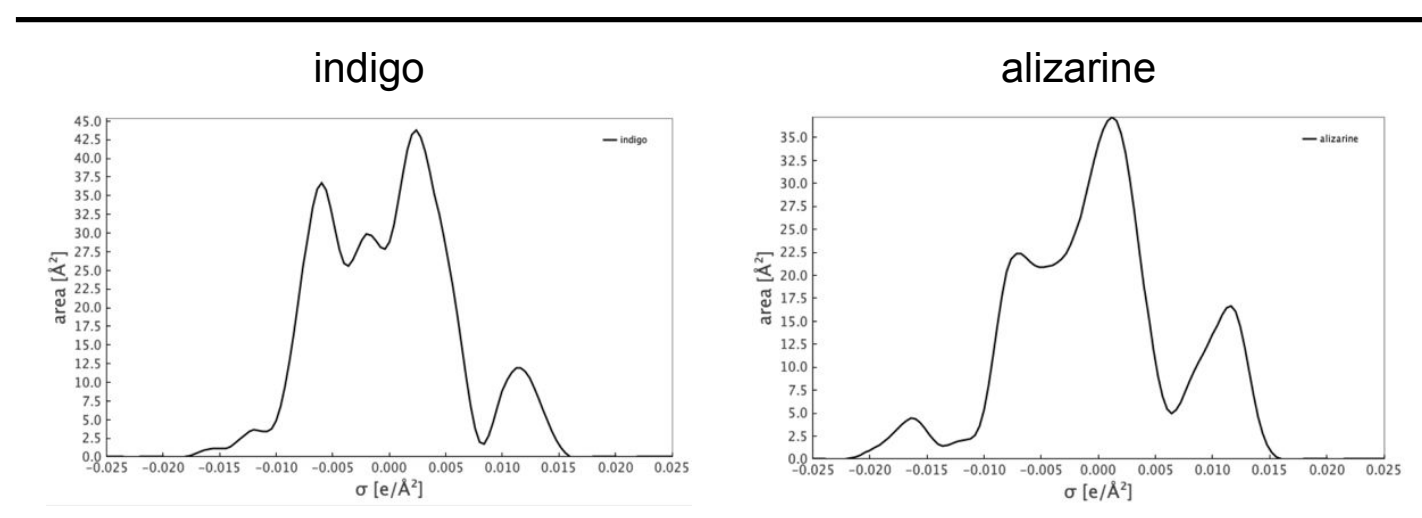

alizarine-1
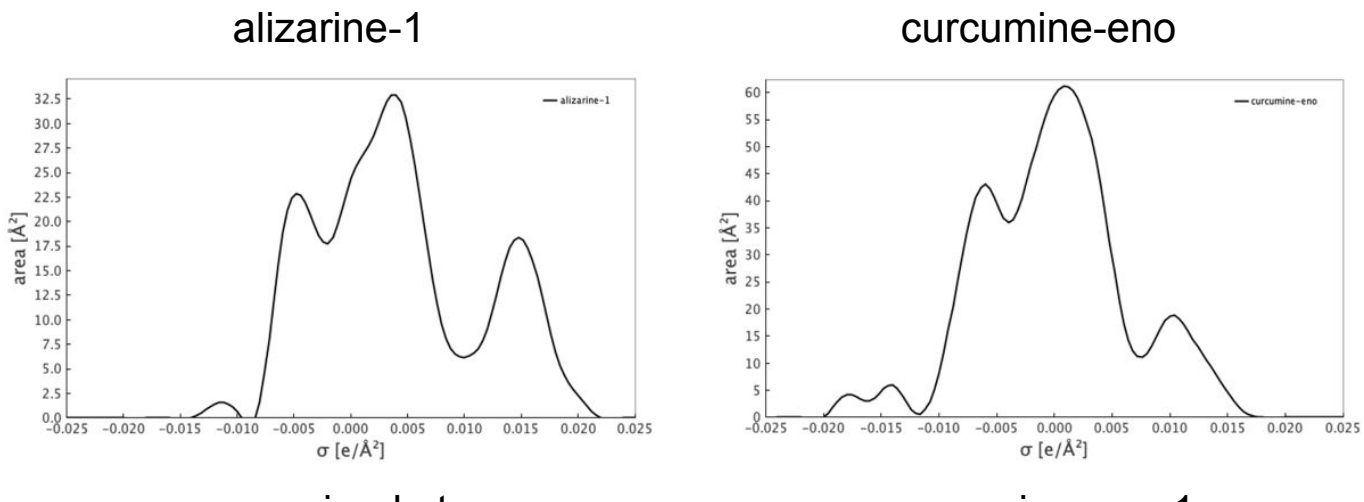

curcumine-keto

\section{curcumine-eno-1}
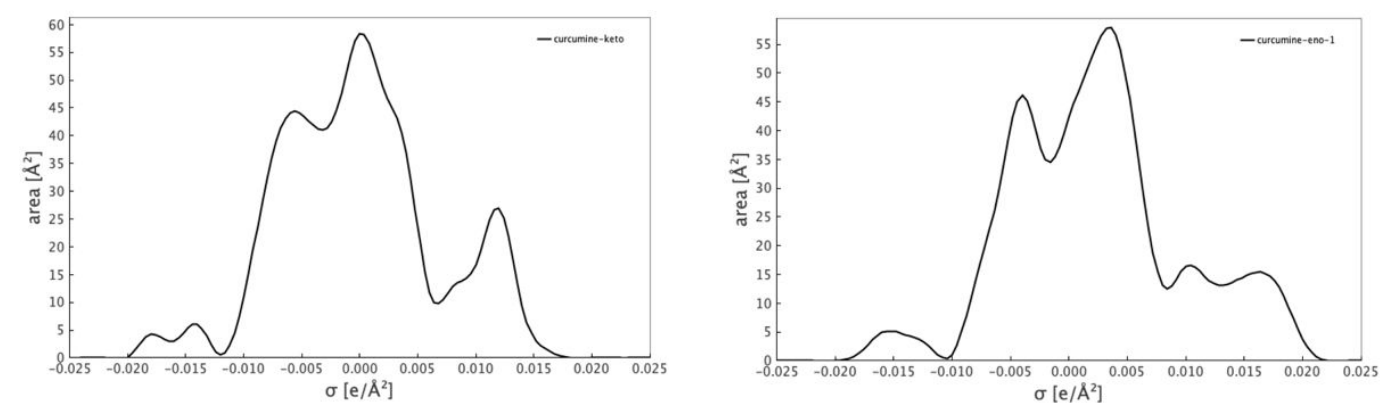

Figure S1. Sigma profiles of the dyes studied in this work. The structures of the dyes are included in Table 1 of the manuscript. 


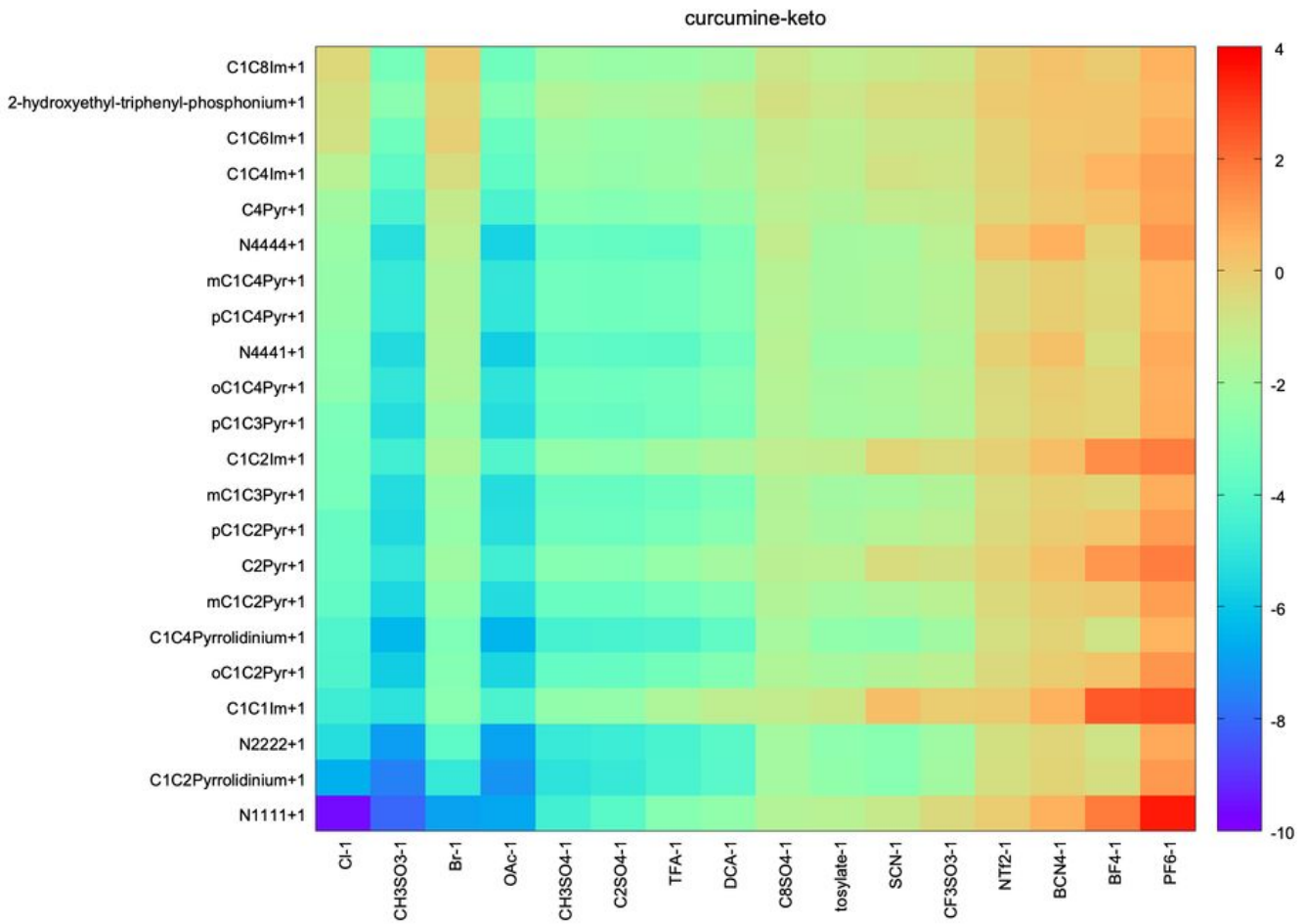

Figure S2. The logarithmic activity coefficients at infinite dilution $\left(\ln \gamma^{\infty}\right)$ of keto tautomer of curcumin in 352 potential ionic liquids calculated by COSMO at $298 \mathrm{~K}$.

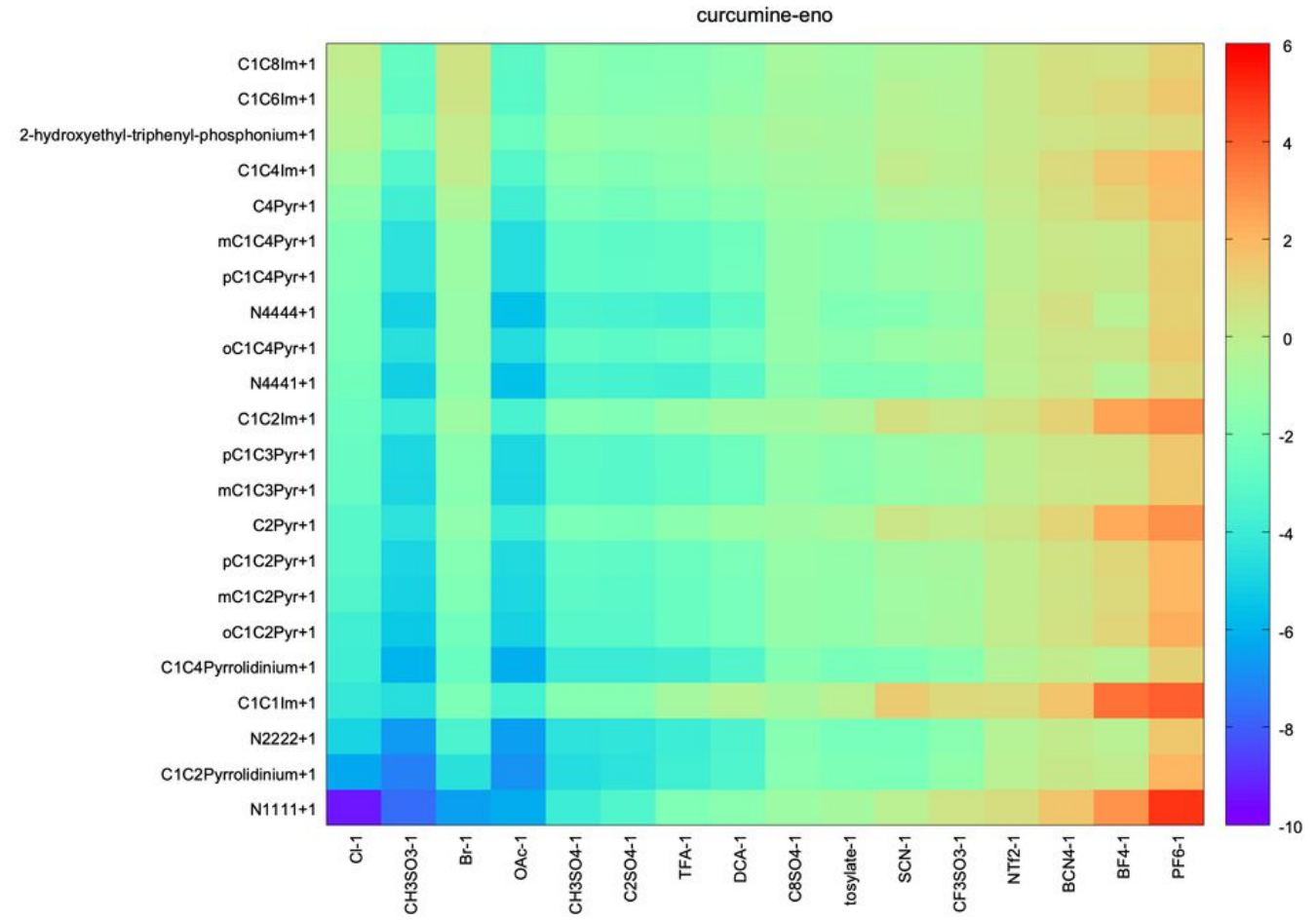

Figure S3. The logarithmic activity coefficients at infinite dilution $\left(\ln \gamma^{\infty}\right)$ of enolic tautomer of curcumin in 352 potential ionic liquids calculated by COSMO at $298 \mathrm{~K}$. 


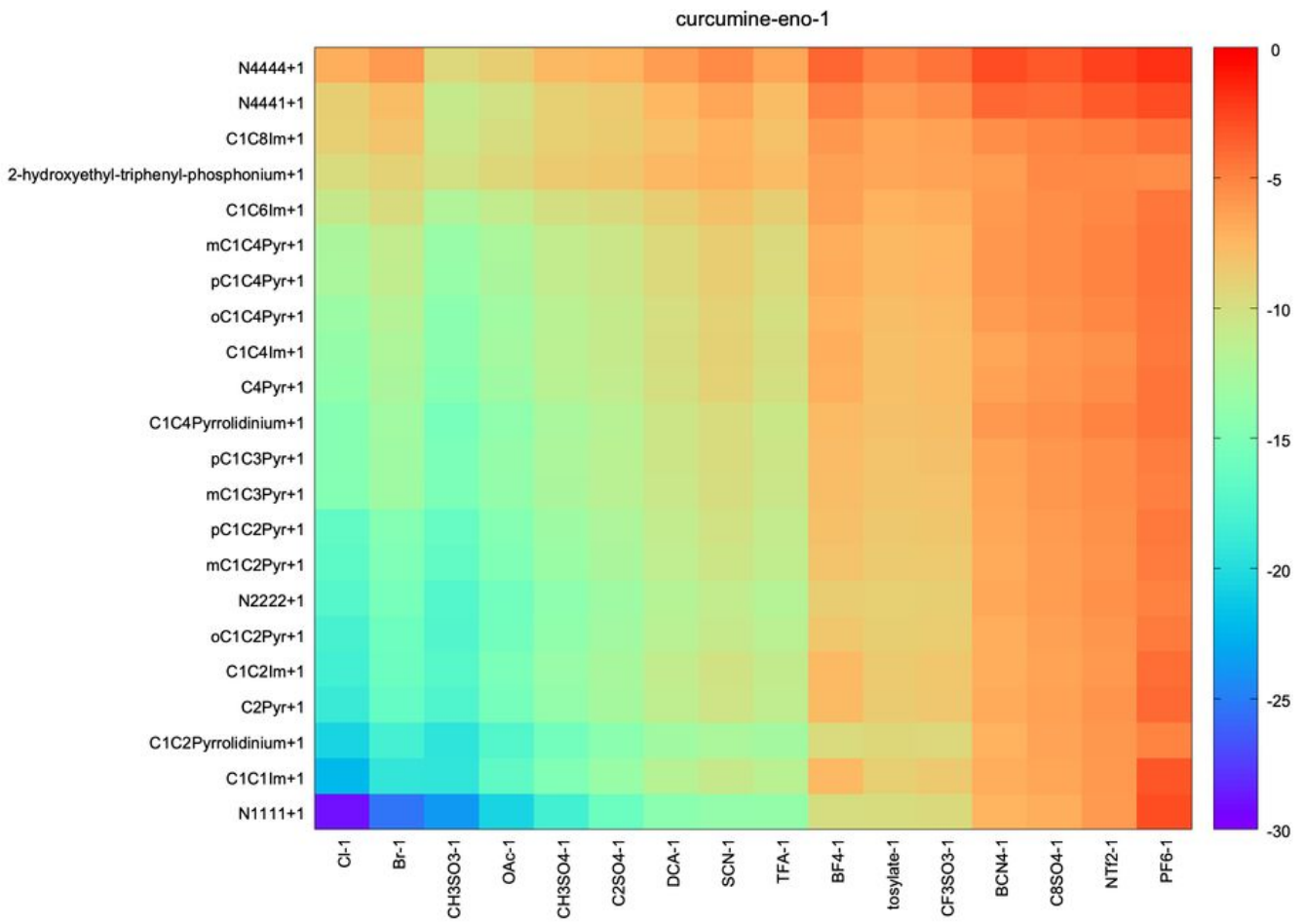

Figure S4. The logarithmic activity coefficients at infinite dilution $\left(\ln \gamma^{\infty}\right)$ of deprotonated curcumin in 352 potential ionic liquids calculated by COSMO at $298 \mathrm{~K}$. 


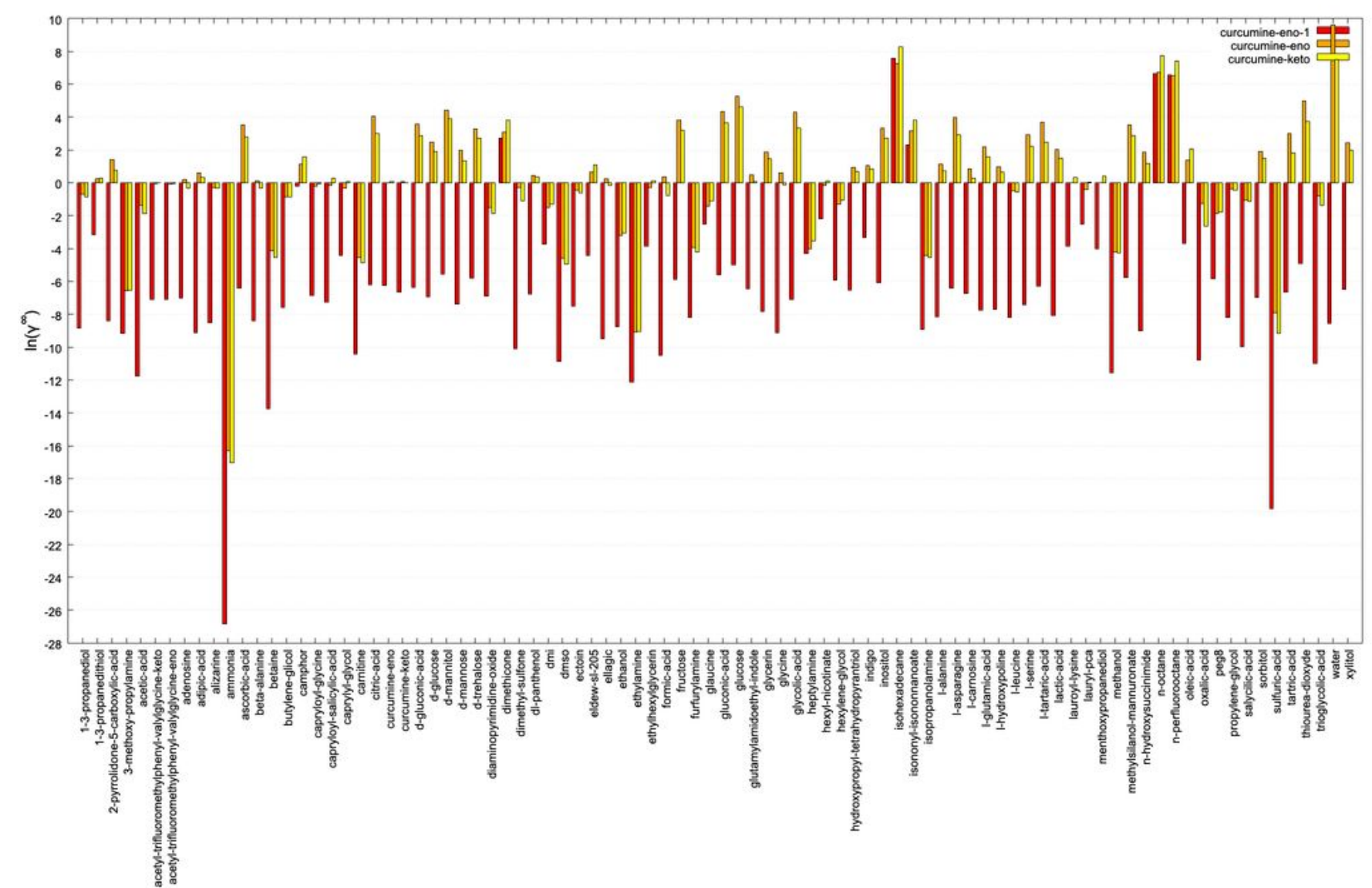

Figure S5. The logarithmic activity coefficients at infinite dilution $\left(\ln \gamma^{\infty}\right)$ of keto (curcumine-keto) and enol (curcumine-eno) tautomers of curcumin, as well as the deprotonated curcumin (curcumine-eno-1) in 88 chemical compounds calculated by COSMO-SAC at $298 \mathrm{~K}$. 
Table S1. Experimental densities of the ionic solvents.

\begin{tabular}{ccccc}
\hline & \multicolumn{4}{c}{$\rho / \mathrm{g} \mathrm{cm}^{3}$} \\
\cline { 2 - 5 } $\mathrm{T} /{ }^{\circ} \mathrm{C}$ & {$\left[\mathrm{C}_{2} \mathrm{C}_{1}\right.$ Im] $\left[\mathrm{EtSO}_{4}\right]$} & $\begin{array}{c}\text { 1-ammonium-2- } \\
\text { propanol lactate } \\
+50 \% \mathrm{H}_{2} \mathrm{O}\end{array}$ & $\begin{array}{c}\text { Betaine : } \\
\text { glycolic } \\
\text { acid }(1: 2)\end{array}$ & $\begin{array}{c}{\left[\mathrm{N}_{4444]}\right][\mathrm{OAc}]} \\
\text { lactic acid } \\
(1: 3)\end{array}$ \\
\hline 25.001 & 1.23612 & 1.10881 & 1.28363 & 1.03622 \\
30.000 & 1.23268 & 1.10612 & 1.27972 & 1.03248 \\
40.000 & 1.22596 & 1.10048 & 1.27299 & 1.02542 \\
60.000 & 1.21266 & 1.08844 & 1.25932 & 1.01111 \\
70.000 & 1.20613 & 1.08213 & 1.25245 & 1.00405 \\
\hline
\end{tabular}

Table S2. Experimental viscosities of the ionic solvents.

\begin{tabular}{|c|c|c|c|c|}
\hline \multirow[b]{2}{*}{$\mathrm{T} /{ }^{\circ} \mathrm{C}$} & \multicolumn{4}{|c|}{$\eta / \mathrm{mPas}$} \\
\hline & {$\left[\mathrm{C}_{2} \mathrm{C}_{1} \mathrm{Im}\right]\left[\mathrm{EtSO}_{4}\right]$} & $\begin{array}{l}\text { 1-ammonium-2- } \\
\text { propanol lactate } \\
+50 \% \mathrm{H}_{2} \mathrm{O}\end{array}$ & $\begin{array}{l}\text { Betaine : } \\
\text { glycolic } \\
\text { acid }(1: 2)\end{array}$ & $\begin{array}{c}{\left[\mathrm{N}_{4444}\right][\mathrm{OAc}]} \\
\text { lactic acid } \\
(1: 3)\end{array}$ \\
\hline 25.001 & 94.256 & 9.1573 & 2998.4 & 358.43 \\
\hline 30.000 & 97.101 & 7.5493 & 1891.6 & 242.28 \\
\hline 40.000 & 67.968 & 5.3328 & 817.76 & 120.08 \\
\hline 60.000 & 34.659 & 3.0376 & 213.93 & 39.611 \\
\hline 70.000 & 25.974 & 2.4081 & 123.95 & 25.377 \\
\hline
\end{tabular}
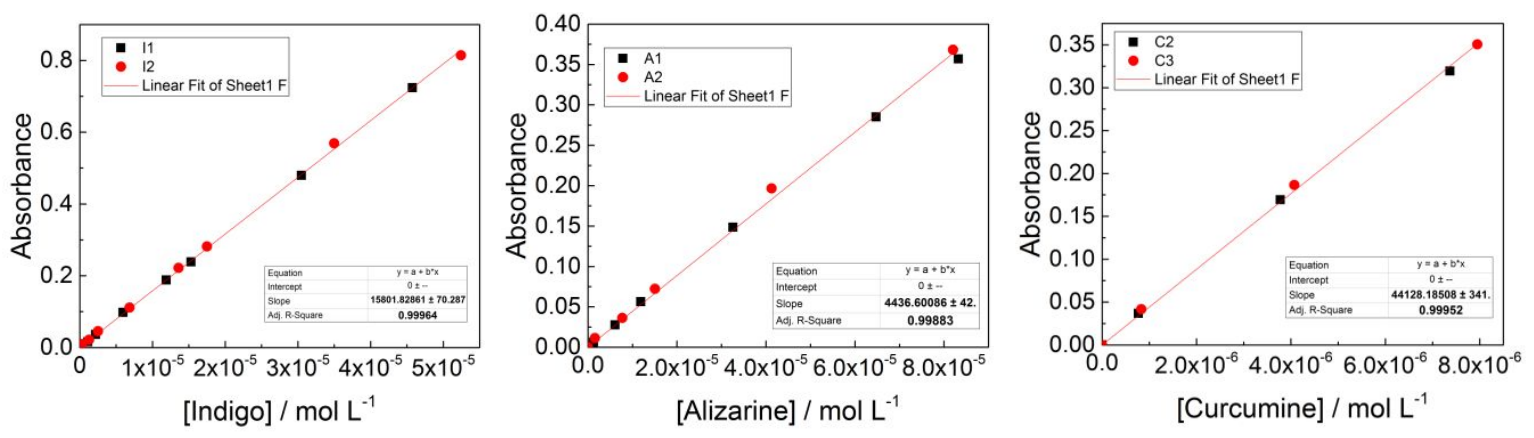

Figure S6. Calibration curve of indigo (left, $\varepsilon_{611.7 \mathrm{~nm}}=15801.8 \mathrm{~L} \mathrm{~mol}^{-1}$ ), alizarin (middle, $\varepsilon_{437 \mathrm{~nm}}=4436.6 \mathrm{~L} \mathrm{~mol}^{-1}$ ) and curcumin (right, $\varepsilon_{434 \mathrm{~nm}}=44128.2 \mathrm{~L} \mathrm{~mol}^{-1}$ ) in DMSO (for indigo) or DMSO / $0.5 \% \mathrm{H}_{2} \mathrm{SO}_{4}$ (for alizarin and curcumin) at room temperature ( 298K). 


\section{References}

1 R. de P. Soares, G. B. Flôres, V. B. Xavier, E. N. Pelisser, F. Ferrarini and P. B. Staudt, Ivpp/sigma: LVPP sigma-profile database (18.07), github.com/lvpp/sigma.

2 F. Ferrarini, G. B. Flôres, A. R. Muniz and R. P. de Soares. An open and extensible sigma-profile database for COSMO-based models. AIChE J., 2018, 64, 3443-3455.

3 R. P. Gerber and R. P. Soares. Assessing the reliability of predictive activity coefficient models for molecules consisting of several functional groups. Brazilian J. Chem. Eng., 2013, 30, 1-11.

4 R. P. Gerber and R. de P. Soares. Prediction of Infinite-Dilution Activity Coefficients Using UNIFAC and COSMO-SAC Variants. Ind. Eng. Chem. Res., 2010, 49, 7488-7496.

5 A. P. Abbott, R. C. Harris, K. S. Ryder, C. D'Agostino, L. F. Gladden and M. D. Mantle. Glycerol eutectics as sustainable solvent systems. Green Chem., 2011, 13, 82-90.

6 C. Ruß and B. König. Low melting mixtures in organic synthesis - an alternative to ionic liquids? Green Chem., 2012, 14, 2969-2982.

7 L. A. Polette-Niewold, F. S. Manciu, B. Torres, M. Alvarado and R. R. Chianelli. Organic/inorganic complex pigments: Ancient colors Maya Blue. J. Inorg. Biochem., 2007, 101, 1958-1973.

8 C. Ma, H. Li, Y. Yang, D. Li and Y. Liu. TD-DFT study on electron transfer mobility and intramolecular hydrogen bond of substituted indigo derivatives. Chem. Phys. Lett., 2015, 638, 72-77.

9 N. Jiwalak, R. Daengngern, T. Rungrotmongkol, S. Jungsuttiwong, S. Namuangruk, N. Kungwan and S. Dokmaisrijan. A spectroscopic study of indigo dye in aqueous solution: A combined experimental and TD-DFT study. $J$. Lumin., 2018, 204, 568-572.

10 N. D. Bernardino, V. R. L. Constantino and D. L. A. de Faria. Probing the Indigo Molecule in Maya Blue Simulants with Resonance Raman Spectroscopy. J. Phys. Chem. C, 2018, 122, 11505-11515. 
11 N. K. Bhatia, S. Kishor, N. Katyal, P. Gogoi, P. Narang and S. Deep. Effect of $\mathrm{pH}$ and temperature on conformational equilibria and aggregation behaviour of curcumin in aqueous binary mixtures of ethanol. RSC Adv., 2016, 6, 103275103288.

12 S. Anjomshoa, M. Namazian and M. R. Noorbala. The Effect of Solvent on Tautomerism, Acidity and Radical Stability of Curcumin and Its Derivatives Based on Thermodynamic Quantities. J. Solution Chem., 2016, 45, 1021-1030.

13 J.-P. Belieres and C. A. Angell. Protic Ionic Liquids: Preparation, Characterization, and Proton Free Energy Level Representation. J. Phys. Chem. B, 2007, 111, 4926-4937. 